\title{
Sums of $L$-functions over rational function fields
}

\author{
by \\ Gautam Chinta (New York, NY) and \\ Joel B. Mohler (Bethlehem, PA)
}

1. Introduction. Let $n$ be an integer greater than or equal to $2, \mathbb{F}_{q}$ the finite field with $q$ elements, and $K=\mathbb{F}_{q}(t)$ the rational function field. The main result of this paper is the explicit computation of an infinite sum of $L$-functions associated to $n$th order Hecke characters of $K$. The infinite sums we consider are examples of double Dirichlet series in two complex variables, and can be written as power series in $q^{-s}$ and $q^{-w}$. In fact it will turn out that the series we construct will be rational functions in $q^{-s}$ and $q^{-w}$.

These series are function field analogs of the series studied by Friedberg, Hoffstein and Lieman in FHL02. In that paper, working over a number field $F$ containing the $n$th roots of unity, the authors study a double Dirichlet series that is roughly of the form

$$
\sum_{m} L\left(s, \chi_{m}\right)(N m)^{-w},
$$

where the sum is over integral ideals $m$ of $F$, the character $\chi_{m}$ is the $n$th order power residue symbol associated to $m$, and $N m$ denotes the absolute norm. The authors show that this double Dirichlet series has a meromorphic continuation to all $(s, w) \in \mathbb{C}^{2}$ and satisfies a group of functional equations relating it to a second series constructed from Gauss sums. The main ingredients in the proof are the functional equation of $L\left(s, \chi_{m}\right)$, properties of the Fourier coefficients of the metaplectic Eisenstein series on the $n$-fold cover of $\mathrm{GL}_{2}$, and Bochner's tube theorem.

In the case $n=2$, these ideas were applied by Fisher and Friedberg FF04] in the context of a general function field to show the rationality of double Dirichlet series constructed from quadratic $L$-functions. The case $n=2$ is somewhat easier because the Gauss sum arising in the functional

2010 Mathematics Subject Classification: Primary 11F66, 11F68, 11M41; Secondary 11F30, 20F55.

Key words and phrases: Weyl group, multiple Dirichlet series, Gauss sums. 
equation of a quadratic Hecke $L$-series is trivial, and the theory of metaplectic Eisenstein series is not needed.

In this paper, we follow a more elementary method originally introduced in [CFH06] in the case $n=2$. Letting $\left(\frac{d}{m}\right)=\chi_{m}(d)$ denote the $n$th power residue symbol for $m, d$ relatively prime, we exploit the fact that (roughly)

$$
\sum_{\substack{d \in \mathbb{F}_{q}[t] \\ \operatorname{deg} d=k}}\left(\frac{d}{m}\right)
$$

vanishes if $k$ is greater than or equal to the degree of $m$, unless $m$ is a perfect $n$th power. Actually this is not quite true unless we carefully define the residue symbol for $d, m$ having a common factor; the exact statement we use is given in (6.1) and depends on the weighting factors $a(d, m)$ we define below.

If $m$ and $d$ are monic and coprime, then we have the reciprocity law

$$
\left(\frac{m}{d}\right)=\left(\frac{d}{m}\right)
$$

when $q$ is congruent to $1 \bmod 2 n$; see e.g. Rosen [Ros02, Theorem 3.5].

We now describe our results more precisely. We will define two double Dirichlet series, explicitly compute them as rational functions in $q^{-s}, q^{-w}$ and show that they satisfy functional equations that relate them to each other. We begin by defining two multiplicative weighting factors $a(d, m)$ and $b(d, m)$ for pairs of monic polynomials, as in [FHL02. For a monic prime polynomial $\mathfrak{p}$, let

$$
\begin{aligned}
& a\left(\mathfrak{p}^{j}, \mathfrak{p}^{k}\right)= \begin{cases}|\mathfrak{p}|^{(n-1) d / n} & \text { if } d=\min (j, k) \text { and } d \equiv 0(\bmod n), \\
0 & \text { otherwise, }\end{cases} \\
& b\left(\mathfrak{p}^{j}, \mathfrak{p}^{k}\right)= \begin{cases}1 & \text { if } k=0, \\
|\mathfrak{p}|^{k / 2-1}(|\mathfrak{p}|-1) & \text { if } j \geq k, k \equiv 0(\bmod n), k>0, \\
-|\mathfrak{p}|^{k / 2-1} & \text { if } j=k-1, k \equiv 0(\bmod n), k>0, \\
|\mathfrak{p}|^{(k-1) / 2} & \text { if } j=k-1, k \neq 0(\bmod n), k>0, \\
0 & \text { otherwise. }\end{cases}
\end{aligned}
$$

Then define

$$
a(d, m)=\prod_{\substack{\mathfrak{p}^{j}\left\|d \\ \mathfrak{p}^{k}\right\| m}} a\left(\mathfrak{p}^{j}, \mathfrak{p}^{k}\right), \quad b(d, m)=\prod_{\substack{\mathfrak{p}^{j}\left\|d \\ \mathfrak{p}^{k}\right\| m}} b\left(\mathfrak{p}^{j}, \mathfrak{p}^{k}\right) .
$$

Here $|d|$ denotes the norm $q^{\operatorname{deg} d}$.

Let $\mathcal{O}$ denote $\mathbb{F}_{q}[t]$ and $\mathcal{O}_{\text {mon }}$ the set of monic polynomials in $\mathbb{F}_{q}[t]$. Let $\zeta_{\mathcal{O}}(s)$ be the zeta function of the ring $\mathcal{O}$, that is,

$$
\zeta_{\mathcal{O}}(s)=\left(1-q^{1-s}\right)^{-1} \text {. }
$$


The first double Dirichlet series we consider is

$$
Z_{1}(s, w)=\sum_{d, m \in \mathcal{O}_{\text {mon }}} \frac{\chi_{m_{0}}(\hat{d}) a(d, m)}{|m|^{w}|d|^{s}}
$$

where $m_{0}$ is the $n$th power free part of $m$ and $\hat{d}$ is the part of $d$ relatively prime to $m_{0}$. By $n$th power free part of $m$ we mean

$$
\prod_{\substack{\mathfrak{p}^{n \alpha+i} \| m \\ 0 \leq i<n}} \mathfrak{p}^{i}
$$

We show in Section 2 that $Z_{1}$ can be rewritten in terms of $L$-functions:

$$
Z_{1}(s, w)=\sum_{m \in \mathcal{O}_{\text {mon }}} \frac{L\left(s, \chi_{m_{0}}\right)}{|m|^{w}} P(s ; m),
$$

where the $P(s ; m)$ are finite Euler products defined in Proposition 2.1

The second multiple Dirichlet series is built from Gauss sums (see Section 2 for the precise definition of the Gauss sum $\left.g\left(r, \epsilon, \chi_{m}\right)\right)$ as follows:

$$
Z_{2}(s, w)=\zeta_{\mathcal{O}}\left(n w-\frac{n}{2}+1\right) \sum_{d, m \in \mathcal{O}_{\text {mon }}} \frac{g\left(1, \epsilon, \chi_{m_{0}}\right)}{\sqrt{\left|m_{b}\right|}} \frac{\bar{\chi}_{m_{0}}(\hat{d}) b(d, m)}{|m|^{w}|d|^{s}},
$$

where $m_{b}$ is the product of primes in the factorization of the $n$th power free part of $m$. Explicitly, if $m=\prod_{i=1}^{r} \mathfrak{p}_{i}^{e_{i}}$, then

$$
m_{b}=\prod_{\substack{i=1 \\ e_{i} \neq 0(\bmod n)}}^{r} \mathfrak{p}_{i} .
$$

We can now state our main theorems. The first describes a set of functional equations relating $Z_{1}$ and $Z_{2}$. Specifically, define

$$
\begin{aligned}
& Z_{1}\left(s, w ; \delta_{i}\right)=\sum_{\substack{d, m \in \mathcal{O}_{\operatorname{mon}} \\
\operatorname{deg} m \equiv i(\bmod n)}} \frac{\chi_{m_{0}}(\hat{d}) a(d, m)}{|m|^{w}|d|^{s}}, \\
& Z_{2}\left(s, w ; \delta_{i}\right)=\zeta_{\mathcal{O}}\left(n w-\frac{n}{2}+1\right) \sum_{\begin{array}{c}
d, m \in \mathcal{O}_{\operatorname{mon}} \\
\operatorname{deg} m \equiv i(\bmod n)
\end{array}} \frac{g\left(1, \epsilon, \chi_{m_{0}}\right)}{\sqrt{\left|m_{b}\right|}} \frac{\bar{\chi}_{m_{0}}(\hat{d}) b(d, m)}{|m|^{w}|d|^{s}} .
\end{aligned}
$$

THEOREM 1.1. We have the functional equation

$$
Z_{1}\left(s, w ; \delta_{i}\right)= \begin{cases}q^{2 s-1} \frac{1-q^{-s}}{1-q^{s-1}} Z_{2}\left(1-s, w+s-\frac{1}{2} ; \delta_{0}\right) & \text { for } i=0, \\ q^{2 s-1} q^{1 / 2-s} \frac{\bar{\tau}\left(\epsilon^{i}\right)}{\sqrt{q}} Z_{2}\left(1-s, w+s-\frac{1}{2} ; \delta_{i}\right) & \text { for } 0<i<n .\end{cases}
$$

The finite field Gauss sum $\tau\left(\epsilon^{i}\right)$ is defined in the following section. 
This is proved in Section 4 .

The second main theorem is the following:

Theorem 1.2. The double Dirichlet series $Z_{1}$ and $Z_{2}$ are rational functions of $x=q^{-s}$ and $y=q^{-w}$. Explicitly,

$$
Z_{1}(s, w)=\frac{1-q^{2} x y}{(1-q x)(1-q y)\left(1-q^{n+1} x^{n} y^{n}\right)}
$$

and

$$
Z_{2}(s, w)=\frac{1-q^{3 n / 2} x^{n-1} y^{n}+\sum_{i=1}^{n-1}\left(\tau\left(\epsilon^{i}\right) q^{i-1+i / 2} x^{i-1} y^{i}-\tau\left(\epsilon^{i}\right) q^{3 i / 2} x^{i} y^{i}\right)}{(1-q x)\left(1-q^{n / 2+1} y^{n}\right)\left(1-q^{3 n / 2} x^{n} y^{n}\right)} .
$$

This theorem is proved in Section 6 .

We conclude this introduction with some remarks to put our results in the larger context of Weyl group multiple Dirichlet series.

The general theory of Weyl group multiple Dirichlet series was introduced in $\left[\mathrm{BBC}^{+} 06\right]$ in order to unify and extend several constructions which had previously been studied. In particular the series $Z_{1}$ and $Z_{2}$ of the present paper are expected to be $n-2$-fold residues of the $n$-variable Weyl group multiple Dirichlet series associated to the root system $A_{n}$. Brubaker and Bump [BB06] have verified this in the cubic case $n=3$. This case is manageable because, thanks to Patterson Pat77a, Pat77b, we have a complete understanding of the Fourier coefficients of the theta function on the 3-fold metaplectic cover of $\mathrm{SL}_{2}$ which arise when we take the residues of the Dirichlet series constructed from cubic Gauss sums.

For $n>3$ the precise nature of the coefficients of the $n$-fold cover theta functions remains mysterious. Nevertheless, there is much evidence in favor of the expectation that the two series constructed by Friedberg, Hoffstein and Lieman coincide with a multiresidue of a Weyl group multiple Dirichlet series. Indeed, one of the motivations of this paper is to lay the groundwork for investigating this question for $n>3$ by explicitly computing and comparing the relevant multiple Dirichlet series in the case of the rational functional field. For example, in [Chi08] the first named author has explicitly computed the cubic $A_{3}$ multiple Dirichlet series and checked that residues of this series give the two series in Theorem 1.2 of this paper when $n=3$. See also [CG] for further examples of explicit computations of double Dirichlet series defined over the rational function field.

Finally we point out a curious connection between the series $Z_{1}, Z_{2}$ of Theorem 1.2 and their $\mathfrak{p}$-parts. Define the following generating series $H_{1}, H_{2}$ 
constructed from the respective $\mathfrak{p}$-parts of $Z_{1}$ and $Z_{2}$ :

$$
\begin{aligned}
& H_{1}(X, Y)=\sum_{j, k \geq 0} a\left(\mathfrak{p}^{j}, \mathfrak{p}^{k}\right) X^{j} Y^{k}, \\
& H_{2}(X, Y)=\left(1-|\mathfrak{p}|^{n / 2-1} Y^{n}\right)^{-1} \sum_{j, k \geq 0} b\left(\mathfrak{p}^{j}, \mathfrak{p}^{k}\right) \frac{g\left(1, \epsilon, \chi_{\mathfrak{p}^{k}}\right)}{\sqrt{\left|\mathfrak{p}_{b}^{k}\right|}} X^{j} Y^{k},
\end{aligned}
$$

where $X=|\mathfrak{p}|^{-s}$ and $Y=|\mathfrak{p}|^{-w}$. We will prove

$$
\begin{aligned}
H_{1}(X, Y) & =\frac{1-X Y}{(1-X)(1-Y)\left(1-|\mathfrak{p}|^{n-1} X^{n} Y^{n}\right)} \\
H_{2}(X, Y) & =\frac{1-|\mathfrak{p}|^{n / 2-1} X^{n-1} Y^{n}+\sum_{i=1}^{n-1} \frac{g\left(1, \epsilon^{i}, \chi_{\mathfrak{p}}\right)}{\sqrt{|\mathfrak{p}|}} X^{i-1} Y^{i}|\mathfrak{p}|^{i-1 / 2}(1-X)}{(1-X)\left(1-|\mathfrak{p}|^{n / 2-1} Y^{n}\right)\left(1-|\mathfrak{p}|^{n / 2} X^{n} Y^{n}\right)}
\end{aligned}
$$

Note that the substitutions

$$
\begin{aligned}
X & \rightarrow q x, \\
Y & \rightarrow q y, \\
|\mathfrak{p}| & \rightarrow 1 / q, \\
g\left(1, \epsilon^{i}, \mathfrak{p}\right) / \sqrt{|\mathfrak{p}|} & \rightarrow \tau\left(\epsilon^{i}\right) / \sqrt{q} \quad \text { for } 1 \leq i \leq r
\end{aligned}
$$

transform $H_{i}$ into $Z_{i}$ for $i=1,2$. This similarity between a rational function field multiple Dirichlet series and its $\mathfrak{p}$-part seems to hold in a much wider context, see e.g. [CFH06, Chi08].

2. Gauss sums and $L$-functions. In this section we will define the Gauss sums and $L$-functions that are the constituents of our double Dirichlet series. We will mostly follow the notation of Patterson [Pat07] but with some adjustments to facilitate comparison with [FHL02.

As in the introduction, $K$ is the rational function field $\mathbb{F}_{q}(t)$ with polynomial ring $\mathcal{O}=\mathbb{F}_{q}[t]$. We let $\mathcal{O}_{\text {mon }}$ denote the subset of $\mathcal{O}$ consisting of monic polynomials and let $K_{\infty}=\mathbb{F}_{q}((t))$ denote the field of Laurent series in $t^{-1}$. Let $\mu_{n}=\left\{a \in \mathbb{F}_{q}: a^{n}=1\right\}$ and let $\chi: \mathbb{F}_{q}^{\times} \rightarrow \mu_{n}$ be the character $a \mapsto a^{(q-1) / n}$.

In order to define Gauss sums we first need an additive character on $K_{\infty}$. Let $e_{0}$ be a nontrivial additive character on the prime field $\mathbb{F}$ of $\mathbb{F}_{q}$. Use this to define a character $e_{\star}$ of $\mathbb{F}_{q}$ by $e_{\star}(a)=e_{0}\left(\operatorname{Tr}_{\mathbb{F}_{q} / \mathbb{F}} a\right)$. Let $\omega$ be the global differential $d x / x^{2}$. Finally define the character $e$ of $K_{\infty}$ by $e(y)=$ $e_{\star}\left(\operatorname{Res}_{\infty}(\omega y)\right)$ for $y \in K_{\infty}$. Note that

$$
\{y \in K: e \mid y \mathcal{O}=1\}=\mathcal{O} .
$$


As in Section 1, for any $c \in \mathcal{O}$, we will use $c_{0}$ to indicate the $n$th power free part of $c$ and $c_{b}$ for the product of primes in the factorization of $c_{0}$. Fix an embedding $\epsilon$ from the $n$th roots of unity of $\mathbb{F}_{q}$ to $\mathbb{C}^{\times}$. For $r, c \in \mathcal{O}$ we define the Gauss sum

$$
g\left(r, \epsilon, \chi_{c}\right)=\sum_{y \bmod c_{b}} \epsilon\left(\left(\frac{y}{c}\right)\right) e\left(\frac{r y}{c_{b}}\right) .
$$

We also need the Gauss sums associated to the finite field $\mathbb{F}_{q}$. These are defined by

$$
\tau(\epsilon)=\sum_{j \in \mathbb{F}_{q}} \epsilon\left(j^{(q-1) / n}\right) e_{0}(j) .
$$

For $m$ monic, we define the $L$-function associated to $\chi_{m}$ by

$$
L\left(s, \chi_{m}\right)=\sum_{d \in \mathcal{O}_{\text {mon }}} \chi_{m}(d)|d|^{-s} .
$$

When $m$ is $n$th power free, the $L$-function satisfies a functional equation that we will describe now. Denote the conductor of the character $\chi_{m}$ by cond $\chi_{m}$. Thus

$$
\left|\operatorname{cond} \chi_{m}\right|= \begin{cases}\left|m_{b}\right|, & \operatorname{deg} m \equiv 0(\bmod n), \\ q\left|m_{b}\right|, & \operatorname{deg} m \not \equiv 0(\bmod n) .\end{cases}
$$

Then the completed $L$-function

$$
L^{*}\left(s, \chi_{m}\right)= \begin{cases}\frac{1}{1-q^{-s}} L\left(s, \chi_{m}\right), & \operatorname{deg} m \equiv 0(\bmod n), \\ L\left(s, \chi_{m}\right), & \operatorname{deg} m \neq \equiv(\bmod n)\end{cases}
$$

satisfies the functional equation

$$
L^{*}\left(s, \chi_{m}\right)=q^{2 s-1}\left|\operatorname{cond} \chi_{m}\right|^{1 / 2-s} \frac{g^{*}\left(1, \epsilon, \chi_{m}\right)}{\left|\operatorname{cond} \chi_{m}\right|^{1 / 2}} L^{*}\left(1-s, \bar{\chi}_{m}\right)
$$

where

$$
g^{*}\left(1, \epsilon, \chi_{m}\right)= \begin{cases}g\left(1, \epsilon, \chi_{m}\right), & \operatorname{deg} m \equiv 0(\bmod n), \\ \bar{\tau}\left(\epsilon^{i}\right) g\left(1, \epsilon, \chi_{m}\right), & \operatorname{deg} m \equiv i \not \equiv 0(\bmod n) .\end{cases}
$$

From the functional equation, we see that $L\left(s, \chi_{m}\right)$ is a polynomial in $q^{-s}$ whose degree is one less than the degree of $m_{b}$, if $m$ is not a perfect $n$th power. If $m=1$ we recover the zeta function

$$
\zeta_{\mathcal{O}}(s)=\sum_{d \in \mathcal{O}_{\text {mon }}}|d|^{-s}=\frac{1}{1-q^{1-s}}
$$

and the functional equation (2.3) holds in this case as well. 
Expanding the components at infinity, we have the following functional equation when $m$ is $n$th power free, $\operatorname{deg} m \equiv i(\bmod n)$ :

$$
\begin{aligned}
& L\left(s, \chi_{m}\right) \\
& = \begin{cases}q^{2 s-1}\left|m_{b}\right|^{1 / 2-s} \frac{g\left(1, \epsilon, \chi_{m}\right)}{\left|m_{b}\right| 1 / 2} \frac{1-q^{-s}}{1-q^{-(1-s)}} L\left(1-s, \bar{\chi}_{m}\right), & i=0, \\
q^{2 s-1}\left(q\left|m_{b}\right|\right)^{1 / 2-s} \frac{\bar{\tau}\left(\epsilon^{i}\right)}{\sqrt{q}} \frac{g\left(1, \epsilon, \chi_{m}\right)}{\left|m_{b}\right|^{1 / 2}} L\left(1-s, \bar{\chi}_{m}\right), & 0<i<n .\end{cases}
\end{aligned}
$$

This functional equation will be used in Section 4 to relate $Z_{1}$ and $Z_{2}$.

We now introduce a modified $L$-function related to (2.1) by inserting the weighting factor $a(d, m)$. Define

$$
L\left(s, \hat{\chi}_{m}\right)=\sum_{d \in \mathcal{O}_{\text {mon }}} \frac{\chi_{m_{0}}(\hat{d}) a(d, m)}{|d|^{s}},
$$

where $\hat{d}$ is the part of $d$ relatively prime to $m_{0}$. Since the weighting function is multiplicative, $L\left(s, \hat{\chi}_{m}\right)$ is an Euler product,

$$
L\left(s, \hat{\chi}_{m}\right)=\prod_{\substack{\mathfrak{p} \in \mathcal{O}_{\text {mon }} \\ \text { irreducible }}}\left(1+\frac{\chi_{m_{0}}(\hat{\mathfrak{p}}) a(\mathfrak{p}, m)}{|\mathfrak{p}|^{s}}+\frac{\chi_{m_{0}}\left(\hat{\mathfrak{p}}^{2}\right) a\left(\mathfrak{p}^{2}, m\right)}{|\mathfrak{p}|^{2 s}}+\cdots\right) .
$$

Further, since $a(d, m)=1$ when $d$ and $m$ are coprime, this Euler product agrees with the original $L$-function Euler product for all but finitely many places.

We will relate this modified $L$-function $L\left(s, \hat{\chi}_{m}\right)$ to $L\left(s, \chi_{m_{0}}\right)$ and derive a bound on its degree as a polynomial in $q^{-s}$, as long as $m$ is not a perfect $n$th power. These properties are given in the following proposition:

Proposition 2.1. We have

$$
L\left(s, \hat{\chi}_{m}\right)=L\left(s, \chi_{m_{0}}\right) P(s ; m),
$$

where $P(s ; m)=\prod_{\mathfrak{p}} P_{\mathfrak{p}}(s ; m)$ and $P_{\mathfrak{p}}(s ; m)$ equals

$$
\left\{\begin{array}{l}
\left(1-\chi_{m_{0}}(\mathfrak{p})|\mathfrak{p}|^{-s}\right) \sum_{k=0}^{n \alpha-1} \frac{\chi_{m_{0}}\left(\mathfrak{p}^{k}\right) a\left(\mathfrak{p}^{n \alpha}, \mathfrak{p}^{k}\right)}{|\mathfrak{p}|^{k s}}+|\mathfrak{p}|^{-n \alpha s}|\mathfrak{p}|^{(n-1) \alpha} \quad \text { if } \mathfrak{p} \nmid m_{0}, \\
\sum_{k=0}^{n \alpha} \frac{a\left(\mathfrak{p}^{n \alpha+i}, \mathfrak{p}^{k}\right)}{|\mathfrak{p}|^{k s}} \quad \text { if } \mathfrak{p}^{i} \| m_{0} \text { and } i \neq 0 .
\end{array}\right.
$$

Here $\alpha$ and $i$ are the unique integers with $0 \leq i<n$ and $\mathfrak{p}^{n \alpha+i} \| m$. In particular, for $m$ not a perfect $n$th power, the degree of $L\left(s, \hat{\chi}_{m}\right)$ as a polynomial in $q^{-s}$ is less than the degree of $m$. 
Proof. Begin with the Euler product

$$
\begin{aligned}
L\left(s, \hat{\chi}_{m}\right) & =\prod_{\mathfrak{p}} \sum_{k=0}^{\infty} \frac{\chi_{m_{0}}\left(\hat{\mathfrak{p}}^{k}\right) a\left(m, \mathfrak{p}^{k}\right)}{|\mathfrak{p}|^{k s}} \\
& =\prod_{\mathfrak{p}^{n \alpha} \| m} \sum_{k=0}^{\infty} \frac{\chi_{m_{0}}\left(\mathfrak{p}^{k}\right) a\left(\mathfrak{p}^{n \alpha}, \mathfrak{p}^{k}\right)}{|\mathfrak{p}|^{k s}} \times \prod_{\substack{\mathfrak{p}^{n \alpha+i} \| m \\
0<i<n}} \sum_{k=0}^{\infty} \frac{a\left(\mathfrak{p}^{n \alpha+i}, \mathfrak{p}^{k}\right)}{|\mathfrak{p}|^{k s}} .
\end{aligned}
$$

For primes $\mathfrak{p}$ with $i=0$ - that is, $\mathfrak{p} \nmid m_{0}$ and $\mathfrak{p}^{n \alpha} \| m$, say-it follows from (1.2) that the tails of the sum are a geometric series with common ratio $\chi_{m_{0}}(\mathfrak{p})|\mathfrak{p}|^{-s}$. Thus for such $\mathfrak{p}$ the $\mathfrak{p}$-part is

$$
\sum_{k=0}^{n \alpha-1} \frac{\chi_{m_{0}}\left(\mathfrak{p}^{k}\right) a\left(\mathfrak{p}^{n \alpha}, \mathfrak{p}^{k}\right)}{|\mathfrak{p}|^{k s}}+\frac{|\mathfrak{p}|^{-n \alpha s}|\mathfrak{p}|^{(n-1) \alpha}}{1-\chi_{m_{0}}(\mathfrak{p})|\mathfrak{p}|^{-s}}=\left(1-\chi_{m_{0}}(\mathfrak{p})|\mathfrak{p}|^{-s}\right)^{-1} P_{\mathfrak{p}}(s ; m),
$$

where

$$
\begin{aligned}
& P_{\mathfrak{p}}(s ; m) \\
& =\sum_{k=0}^{n \alpha-1} \frac{\chi_{m_{0}}\left(\mathfrak{p}^{k}\right) a\left(\mathfrak{p}^{n \alpha}, \mathfrak{p}^{k}\right)}{|\mathfrak{p}|^{k s}}\left(1-\chi_{m_{0}}(\mathfrak{p})|\mathfrak{p}|^{-s}\right)+|\mathfrak{p}|^{-n \alpha s}|\mathfrak{p}|^{(n-1) \alpha} .
\end{aligned}
$$

For primes such that $\mathfrak{p}^{i} \| m_{0}$ with $0<i<n$, it follows from 1.2 that $a\left(\mathfrak{p}^{n \alpha+i}, \mathfrak{p}^{k}\right)=0$ for $k>n \alpha$, so the $\mathfrak{p}$-part is a finite sum

$$
P_{\mathfrak{p}}(s ; m)=\sum_{k=0}^{n \alpha} \frac{a\left(\mathfrak{p}^{n \alpha+i}, \mathfrak{p}^{k}\right)}{|\mathfrak{p}|^{k s}} .
$$

Thus

$$
L\left(s, \hat{\chi}_{m}\right)=L\left(s, \chi_{m_{0}}\right) P(s ; m)
$$

as claimed. The bound on the degree of $L\left(s, \hat{\chi}_{m}\right)$ follows from the bound on the degree of $L\left(s, \chi_{m_{0}}\right)$ for $m_{0} \neq 1$ and the degrees of the $P_{p}(s ; m)$.

3. Functional equation $H_{1} \rightarrow H_{2}$. Recall that the generating series $H_{1}(X, Y)$ and $H_{2}(X, Y)$ of 1.9$)$ define the $p$-parts of $Z_{1}$ and $Z_{2}$, respectively. We describe the functional equations relating $H_{1}(X, Y)$ and $H_{2}(X, Y)$. These will be used to prove the global functional equation relating $Z_{1}$ to $Z_{2}$.

The functional equations are a direct consequence of the following proposition:

Proposition 3.1. The generating series $H_{1}(X, Y)$ and $H_{2}(X, Y)$ are rational functions of $X$ and $Y$. Explicitly,

$$
H_{1}(X, Y)=\frac{1-X Y}{(1-X)(1-Y)\left(1-|\mathfrak{p}|^{n-1} X^{n} Y^{n}\right)}
$$


and

$$
\begin{aligned}
& H_{2}(X, Y) \\
& \quad \begin{array}{l}
1-|\mathfrak{p}|^{n / 2-1} X^{n-1} Y^{n}+\sum_{i=1}^{n-1} \frac{g\left(1, \epsilon^{i}, \chi_{\mathfrak{p}}\right)}{\sqrt{|\mathfrak{p}|}} X^{i-1} Y^{i}|\mathfrak{p}|^{(i-1) / 2}(1-X) \\
(1-X)\left(1-|\mathfrak{p}|^{n / 2-1} Y^{n}\right)\left(1-|\mathfrak{p}|^{n / 2} X^{n} Y^{n}\right)
\end{array}
\end{aligned}
$$

Proof. Equation (3.1) is obvious from the definition 1.2 of the $a\left(\mathfrak{p}^{k}, \mathfrak{p}^{l}\right)$. The evaluation of $H_{2}(X, Y)$ is simply a matter of recognizing geometric series.

From the definitions of $b\left(\mathfrak{p}^{j}, \mathfrak{p}^{k}\right)$ in $(1.3)$ and $H_{2}$ in $(1.9)$, we have

$$
\begin{aligned}
\left(1-|\mathfrak{p}|^{n / 2-1} Y^{n}\right) & H_{2}(X, Y) \\
= & \sum_{j=0}^{\infty} \frac{g\left(1, \epsilon, \chi_{\mathfrak{p}^{0}}\right)}{\sqrt{\left|\mathfrak{p}_{b}^{0}\right|}} X^{j} Y^{0} \\
& +\sum_{\alpha=1}^{\infty} \sum_{j=n \alpha}^{\infty}|\mathfrak{p}|^{n \alpha / 2-1}(|\mathfrak{p}|-1) \frac{g\left(1, \epsilon, \chi_{\mathfrak{p}^{n \alpha}}\right)}{\sqrt{\left|\mathfrak{p}_{b}^{n \alpha}\right|}} X^{j} Y^{n \alpha} \\
& +\sum_{\alpha=1}^{\infty}-|\mathfrak{p}|^{n \alpha / 2-1} \frac{g\left(1, \epsilon, \chi_{\left.\mathfrak{p}^{n \alpha}\right)}\right.}{\sqrt{\left|\mathfrak{p}_{b}^{n \alpha}\right|} X^{n \alpha-1} Y^{n \alpha}} \\
& +\sum_{\alpha=0}^{\infty} \sum_{i=1}^{n-1}|\mathfrak{p}|^{(n \alpha+i-1) / 2} \frac{g\left(1, \epsilon, \chi_{\mathfrak{p}^{n \alpha+i}}\right)}{\sqrt{\left|\mathfrak{p}_{b}^{n \alpha+i}\right|}} X^{n \alpha+i-1} Y^{n \alpha+i}
\end{aligned}
$$

Evaluating the geometric series yields

$$
\begin{aligned}
\left(1-|\mathfrak{p}|^{n / 2-1} Y^{n}\right) H_{2}(X, Y) & \\
= & \frac{1}{1-X}+\frac{|\mathfrak{p}|^{n / 2-1}(|\mathfrak{p}|-1) X^{n} Y^{n}}{(1-X)\left(1-|\mathfrak{p}|^{n / 2} X^{n} Y^{n}\right)} \\
& +\frac{-|\mathfrak{p}|^{n / 2-1} X^{n-1} Y^{n}}{1-|\mathfrak{p}|^{n / 2} X^{n} Y^{n}}+\sum_{i=1}^{n-1} \frac{\frac{g\left(1, \epsilon^{i}, \chi_{\mathfrak{p}}\right)}{\sqrt{|\mathfrak{p}|}}|\mathfrak{p}|^{(i-1) / 2} X^{i-1} Y^{i}}{\left(1-|\mathfrak{p}|^{n / 2} X^{n} Y^{n}\right)}
\end{aligned}
$$

Equation (3.2) follows by rewriting (3.3). 
For $0 \leq i<n$, define

$$
\begin{aligned}
H_{1}\left(X, Y ; \delta_{i}\right) & =\sum_{\substack{j, k \geq 0 \\
k \equiv i(\bmod n)}} a\left(\mathfrak{p}^{j}, \mathfrak{p}^{k}\right) X^{j} Y^{k}, \\
H_{2}\left(X, Y ; \delta_{i}\right)=\left(1-|\mathfrak{p}|^{n / 2-1} Y^{n}\right)^{-1} & \sum_{\substack{j, k \geq 0 \\
k \equiv i(\bmod n)}} b\left(\mathfrak{p}^{j}, \mathfrak{p}^{k}\right) \\
& \times \frac{g\left(1, \epsilon, \chi_{\mathfrak{p}^{k}}\right)}{\sqrt{\left|\mathfrak{p}_{b}^{k}\right|}} \bar{\chi}_{\mathfrak{p}^{k}}\left(\hat{\mathfrak{p}}^{j}\right) X^{j} Y^{k} .
\end{aligned}
$$

We have shown in Proposition 3.1 that $H_{1}$ and $H_{2}$ are both rational functions in $|\mathfrak{p}|^{-s}$ and $|\mathfrak{p}|^{-w}$ and it is clear from this proposition that

$$
\begin{aligned}
& H_{1}\left(X, Y ; \delta_{i}\right)= \begin{cases}\frac{1-X Y^{n}}{(1-X)\left(1-Y^{n}\right)\left(1-|\mathfrak{p}|^{n-1} X^{n} Y^{n}\right)}, & i \equiv 0, \\
\frac{(1-X) Y^{i}}{(1-X)\left(1-Y^{n}\right)\left(1-|\mathfrak{p}|^{n-1} X^{n} Y^{n}\right)}, & i \neq 0,\end{cases} \\
& H_{2}\left(X, Y ; \delta_{i}\right)= \begin{cases}\frac{1-|\mathfrak{p}|^{n / 2-1} X^{n-1} Y^{n}}{(1-X)\left(1-|\mathfrak{p}|^{n / 2-1} Y^{n}\right)\left(1-|\mathfrak{p}|^{n / 2} X^{n} Y^{n}\right)}, & i \equiv 0, \\
\frac{g\left(1, \epsilon^{i}, \chi_{\mathfrak{p}}\right)}{\sqrt{|\mathfrak{p}|} X^{i-1} Y^{i}|\mathfrak{p}|^{(i-1) / 2}(1-X)} & \\
\frac{(1-X)\left(1-|\mathfrak{p}|^{n / 2-1} Y^{n}\right)\left(1-|\mathfrak{p}|^{n / 2} X^{n} Y^{n}\right)}{(1-X}, & i \neq 0 .\end{cases}
\end{aligned}
$$

The following theorem establishes a functional equation relating $H_{1}$ to $H_{2}$ :

THEOREM 3.2. We have the functional equation

$$
\begin{aligned}
& H_{1}\left(|\mathfrak{p}|^{-s},|\mathfrak{p}|^{-w} ; \delta_{i}\right) \\
& = \begin{cases}\frac{1-|\mathfrak{p}|^{-(1-s)}}{1-|\mathfrak{p}|^{-s}} H_{2}\left(|\mathfrak{p}|^{-(1-s)},|\mathfrak{p}|^{-(w+s-1 / 2)} ; \delta_{0}\right), & i=0, \\
\frac{\sqrt{|\mathfrak{p}|}}{g\left(1, \epsilon^{i}, \chi_{\mathfrak{p}}\right)}|\mathfrak{p}|^{s-1 / 2} H_{2}\left(|\mathfrak{p}|^{-(1-s)},|\mathfrak{p}|^{-(w+s-1 / 2)} ; \delta_{i}\right), & 0<i<n .\end{cases}
\end{aligned}
$$

Proof. The proof is by a direct computation using equations (3.5) and (3.6).

4. Functional equation $Z_{1} \rightarrow Z_{2}$. There is a set of functional equations relating $Z_{1}$ and $Z_{2}$. These will be described in this section. Define

$$
Q(s ; m)=\frac{P(1-s ; m)}{\left(m / m_{b}\right)^{s-1 / 2}} .
$$


With the expansion of $P$ as an Euler product, we see that $Q$ is also an Euler product supported in the primes dividing $m$ :

$$
\begin{aligned}
Q(s ; m)= & \prod_{\substack{\mathfrak{p}^{n \alpha+i} \| m \\
i=0}} \frac{1}{|\mathfrak{p}|^{n \alpha(s-1 / 2)}} P_{\mathfrak{p}}(1-s ; m) \\
& \times \prod_{\substack{\mathfrak{p}^{n \alpha+i} \| m \\
0<i<n}} \frac{1}{|\mathfrak{p}|^{(n \alpha+i-1)(s-1 / 2)}} P_{\mathfrak{p}}(1-s ; m) .
\end{aligned}
$$

Proposition 4.1. Define

$$
Z_{2}^{\prime}(s, w)=\sum_{m} \frac{g\left(1, \epsilon, \chi_{m_{0}}\right)}{\sqrt{\left|m_{b}\right|}} \frac{L\left(s, \bar{\chi}_{m_{0}}\right) Q(s ; m)}{|m|^{w}} .
$$

We have $Z_{2}^{\prime}=Z_{2}$.

Proof. Define

$$
H_{2}^{\prime}\left(|\mathfrak{p}|^{-s},|\mathfrak{p}|^{-w} ; \delta_{i}\right)= \begin{cases}\left(1-|\mathfrak{p}|^{-s}\right)^{-1} \sum_{k=0}^{\infty} \frac{Q\left(s ; \mathfrak{p}^{n k}\right)}{|\mathfrak{p}|^{n k w}}, & i=0, \\ \frac{g\left(1, \epsilon^{i}, \chi_{\mathfrak{p}}\right)}{\sqrt{|\mathfrak{p}|}} \sum_{k=0}^{\infty} \frac{Q\left(s ; \mathfrak{p}^{n k+i}\right)}{|\mathfrak{p}|^{n k w}}, & 0<i<n .\end{cases}
$$

Then $H_{2}^{\prime}\left(|\mathfrak{p}|^{-s},|\mathfrak{p}|^{-w}\right)=\sum_{i=0}^{n-1} H_{2}^{\prime}\left(|\mathfrak{p}|^{-s},|\mathfrak{p}|^{-w} ; \delta_{i}\right)$ is the $\mathfrak{p}$-part of $Z_{2}^{\prime}$. We will show that $H_{2}^{\prime}$ and $H_{2}$ both satisfy the functional equations with $H_{1}$ shown in Theorem 3.2 and therefore $H_{2}^{\prime}=H_{2}$. The result follows since, for fixed $m$, the $L$-functions $P$ and $Q$ each have Euler products.

As a result of the definition in equation (4.1), the p-parts of $Q$ and $P$ satisfy

$$
P\left(s ; \mathfrak{p}^{n \alpha+i}\right)= \begin{cases}|\mathfrak{p}|^{n \alpha(1 / 2-s)} Q\left(1-s ; \mathfrak{p}^{n \alpha}\right), & i=0, \\ |\mathfrak{p}|^{(n \alpha+i-1)(1 / 2-s)} Q\left(1-s ; \mathfrak{p}^{n \alpha+i}\right), & 0<i<n .\end{cases}
$$

Therefore, we relate $H_{1}$ to $H_{2}^{\prime}$ by

$$
\begin{aligned}
H_{1}\left(|\mathfrak{p}|^{-s},|\mathfrak{p}|^{-w} ; \delta_{0}\right) & =\left(1-|\mathfrak{p}|^{-s}\right)^{-1} \sum_{k=0}^{\infty} \frac{P\left(s ; \mathfrak{p}^{n k}\right)}{|\mathfrak{p}|^{n k w}} \\
& =\left(1-|\mathfrak{p}|^{-s}\right)^{-1} \sum_{k=0}^{\infty} \frac{Q\left(1-s ; \mathfrak{p}^{n k}\right)|\mathfrak{p}|^{n k(1 / 2-s)}}{|\mathfrak{p}|^{n k w}} \\
& =\left(1-|\mathfrak{p}|^{-s}\right)^{-1} \sum_{k=0}^{\infty} \frac{Q\left(1-s ; \mathfrak{p}^{n k}\right)}{|\mathfrak{p}|^{n k(w+s-1 / 2)}} \\
& =\frac{1-|\mathfrak{p}|^{-(1-s)}}{1-|\mathfrak{p}|^{-s}} H_{2}^{\prime}\left(|\mathfrak{p}|^{-(1-s)},|\mathfrak{p}|^{-(w+s-1 / 2)} ; \delta_{0}\right) .
\end{aligned}
$$


This is exactly the functional equation satisfied by $H_{2}$ in Theorem 3.2 A similar computation shows that

$$
H_{1}\left(|\mathfrak{p}|^{-s},|\mathfrak{p}|^{-w} ; \delta_{i}\right)=\frac{\sqrt{|\mathfrak{p}|}}{g\left(1, \epsilon^{i}, \chi_{\mathfrak{p}}\right)}|\mathfrak{p}|^{s-1 / 2} H_{2}\left(|\mathfrak{p}|^{-(1-s)},|\mathfrak{p}|^{-(w+s-1 / 2)} ; \delta_{i}\right)
$$

for $0<i<n$. Thus $H_{2}^{\prime}\left(X, Y ; \delta_{i}\right)=H_{2}\left(X, Y ; \delta_{i}\right)$ for all $0 \leq i<n$ and this completes the proof.

For $0 \leq i<n$, define

$$
\begin{aligned}
& Z_{1}\left(s, w ; \delta_{i}\right)=\sum_{\substack{m \in \mathcal{O}_{\operatorname{mon}} \\
\operatorname{deg} m \equiv i(\bmod n)}} \frac{L\left(s, \chi_{m_{0}}\right) P(s ; m)}{|m|^{w}}, \\
& Z_{2}\left(s, w ; \delta_{i}\right)=\sum_{\substack{m \in \mathcal{O}_{\operatorname{mon}} \\
\operatorname{deg} m \equiv i(\bmod n)}} \frac{g\left(1, \epsilon, \chi_{m_{0}}\right)}{\sqrt{\left|m_{b}\right|}} \frac{L\left(s, \bar{\chi}_{m_{0}}\right) Q(s ; m)}{|m|^{w}} .
\end{aligned}
$$

THEOREM 4.2. We have the functional equation

$$
Z_{1}\left(s, w ; \delta_{i}\right)= \begin{cases}q^{2 s-1} \frac{1-q^{-s}}{1-q^{s-1}} Z_{2}\left(1-s, w+s-\frac{1}{2} ; \delta_{0}\right) & \text { for } i=0, \\ q^{2 s-1} q^{1 / 2-s} \frac{\bar{\tau}\left(\epsilon^{i}\right)}{\sqrt{q}} Z_{2}\left(1-s, w+s-\frac{1}{2} ; \delta_{i}\right) & \text { for } 0<i<n .\end{cases}
$$

Proof. This is a direct computation utilizing the functional equation 2.5) for $L\left(s, \chi_{m_{0}}\right)$.

5. Convolutions. We define a convolution operation $\star$ on rational functions in $x$ and $y$ with power series expansions around the origin. For

$$
A(x, y)=\sum_{j, k \geq 0} a(j, k) x^{j} y^{k} \quad \text { and } \quad B(x, y)=\sum_{j, k \geq 0} b(j, k) x^{j} y^{k},
$$

define

$$
(A \star B)(x, y)=\sum_{j, k \geq 0} a(j, k) b(j, k) x^{j} y^{k} .
$$

We can compute convolutions as the double integral

$$
(A \star B)(x, y)=\left(\frac{1}{2 \pi i}\right)^{2} \iint A(u, v) B\left(\frac{x}{u}, \frac{y}{v}\right) \frac{d u d v}{u v},
$$

where each integral is a counterclockwise circuit of a small circle in the complex plane. (The circle must be small enough that $A(x, y)$ is holomorphic for $x, y$ inside the circle.) We will utilize the residue theorem to compute this contour integral. 
6. Evaluation of $Z_{1}$ and $Z_{2}$. We will now prove Theorem 1.2 . We first establish the identity (1.7); then $(1.8)$ will follow from the functional equation (Theorem 1.1). It follows from Proposition 2.1 that

$$
\sum_{\substack{d \in \mathcal{O}_{\text {mon }} \\ \operatorname{deg} d=k}} \chi_{m_{0}}(\hat{d}) a(d, m)=0
$$

when $\operatorname{deg} m \leq k$ unless $m$ is a perfect $n$th power. To prove (1.7) of Theorem 1.2 , we begin by writing

$$
Z_{1}(s, w)=Z_{a}(s, w)+Z_{a}(w, s)-Z_{b}(s, w)
$$

where

$$
\begin{aligned}
& Z_{a}(s, w)=\sum_{k \geq j \geq 0} \frac{1}{q^{j w} q^{k s}} \sum_{\begin{array}{r}
d, m \in \mathcal{O}_{\text {mon }} \\
\operatorname{deg} m=j \\
\operatorname{deg} d=k
\end{array}} \chi_{m_{0}}(\hat{d}) a(d, m), \\
& Z_{b}(s, w)=\sum_{k \geq 0} \frac{1}{q^{k w} q^{k s}} \sum_{\substack{m \in \mathcal{O}_{\text {mon }} \\
\operatorname{deg} m=j \operatorname{deg} d=k}} \sum_{\substack{\text { mon } \\
\text { deg }}} \chi_{m_{0}}(\hat{d}) a(d, m) .
\end{aligned}
$$

First, note that

$$
\sum_{\substack{m \in \mathcal{O}_{\text {mon }} \\ \operatorname{deg} m=j \operatorname{deg} d=k}} \chi_{\substack{\text { mon } \\ d=k}}(\hat{d}) a(d, m)=\sum_{\substack{m \in \mathcal{O}_{\text {mon }} \\ \operatorname{deg} m=j \operatorname{deg} d=k}} \sum_{\substack{\text { mon } \\ d=k}} \chi_{d_{0}}(\hat{m}) a(m, d) .
$$

When $m$ and $d$ are coprime, the reciprocity law 1.1 guarantees that $\chi_{m_{0}}(\hat{d})$ $=\chi_{d_{0}}(\hat{m})$. Otherwise, when $m$ and $d$ are not coprime, $\chi_{m_{0}}(\hat{d}) \neq \chi_{d_{0}}(\hat{m})$ only when there exists a prime $\mathfrak{p}$ such that $\mathfrak{p} \mid d_{0}$ and $\mathfrak{p} \mid m_{0}$. In this case $a(d, m)=0$. The symmetry $a(d, m)=a(m, d)$ is obvious. This establishes the validity of the decomposition (6.2) of $Z_{1}$.

Now the key observation is that because of equation (6.1), we have

$$
\begin{aligned}
& Z_{a}(s, w)=\sum_{k \geq j \geq 0} \frac{1}{q^{j w} q^{k s}} \sum_{\substack{m \in \mathcal{O}_{\text {mon }} \\
\text { deg } m=j \operatorname{deg} d=k \\
m_{0}=1}} \sum_{\substack{d \in \mathcal{O}_{\text {mon }} \\
d=k}} a(d, m), \\
& Z_{b}(s, w)=\sum_{k \geq 0} \frac{1}{q^{k w} q^{k s}} \sum_{\substack{m \in \mathcal{O}_{\text {mon }} \\
\operatorname{deg} m=k \operatorname{deg} d=k \\
m_{0}=1}} \sum_{\substack{d \in \mathcal{O}_{\text {mon }} \\
\text { d }}} a(d, m),
\end{aligned}
$$

that is, the inner sum vanishes unless $m$ is a perfect $n$th power. This leads us to consider the series

$$
T_{a}(s, w)=\sum_{\substack{m \in \mathcal{O}_{\text {mon }} \\ m_{0}=1}} \sum_{d \in \mathcal{O}_{\text {mon }}} \frac{a(d, m)}{|m|^{w}|d|^{s}}
$$


which has an Euler product

$$
\begin{aligned}
T_{a}(s, w) & =\prod_{\mathfrak{p}} \sum_{j, k \geq 0} \frac{a\left(\mathfrak{p}^{j}, \mathfrak{p}^{n k}\right)}{|\mathfrak{p}|^{n k}|\mathfrak{p}|^{j}} \\
& =\prod_{\mathfrak{p}} \frac{1-|\mathfrak{p}|^{-s-n w}}{\left(1-|\mathfrak{p}|^{-s}\right)\left(1-|\mathfrak{p}|^{-n w}\right)\left(1-|\mathfrak{p}|^{(n-1)-n s-n w}\right)} \\
& =\frac{\zeta(s) \zeta(n w) \zeta(n s+n w-(n-1))}{\zeta(s+n w)} \\
& =\frac{1-q^{1-s-n w}}{\left(1-q^{1-s}\right)\left(1-q^{1-n w}\right)\left(1-q^{n-n s-n w}\right)} \\
& =\frac{1-q x y^{n}}{(1-q x)\left(1-q y^{n}\right)\left(1-q^{n} x^{n} y^{n}\right)}=\tilde{T}_{a}(x, y),
\end{aligned}
$$

with $x=q^{-s}, y=q^{-w}$.

It is clear that

$$
Z_{a}(s, w)=\left(\tilde{T}_{a} \star \tilde{K}_{a}\right)(x, y)
$$

where

$$
\tilde{K}_{a}(x, y)=\frac{1}{(1-x)(1-x y)}=\sum_{j \geq k \geq 0} x^{j} y^{k} .
$$

Compute the convolution in (6.4) by means of the integral in equation (5.1), which we can evaluate using the residue theorem. We find

$$
Z_{a}(s, w)=\frac{1}{\left(1-q^{n+1} x^{n} y^{n}\right)(1-q x)} .
$$

We can compute $Z_{b}$ similarly: let $\tilde{K}_{b}(x, y)=1 /(1-x y)$. Then

$$
Z_{b}(s, w)=\left(\tilde{T}_{a} \star \tilde{K}_{b}\right)(x, y)=\frac{1}{1-q^{n+1} x^{n} y^{n}} .
$$

Putting this all together,

$$
\begin{aligned}
Z_{1}(s, w)= & \frac{1}{\left(1-q^{n+1} x^{n} y^{n}\right)(1-q x)}+\frac{1}{\left(1-q^{n+1} x^{n} y^{n}\right)(1-q y)} \\
& -\frac{1}{1-q^{n+1} x^{n} y^{n}} \\
= & \frac{1-q y+1-q x-(1-q y)(1-q x)}{\left(1-q^{n+1} x^{n} y^{n}\right)(1-q x)(1-q y)} \\
= & \frac{1-q^{2} x y}{\left(1-q^{n+1} x^{n} y^{n}\right)(1-q x)(1-q y)} .
\end{aligned}
$$

This establishes (1.7).

With the rational function for $Z_{1}(s, w)$, we can use the functional equations relating $Z_{1}\left(s, w ; \delta_{i}\right)$ and $Z_{2}\left(s, w ; \delta_{i}\right)$ for $0 \leq i<n$ to evaluate $Z_{2}(s, w)$. 
Expanding the geometric series $1 /(1-q y)$ and collecting terms with the exponent on $y$ congruent to $i \bmod n$, we arrive at

$$
Z_{1}\left(s, w ; \delta_{i}\right)= \begin{cases}\frac{1-q^{n+1} x y^{n}}{(1-q x)\left(1-q^{n} y^{n}\right)\left(1-q^{n+1} x^{n} y^{n}\right)}, & i=0, \\ \frac{\left(q^{i}-q^{i+1} x\right) y^{i}}{(1-q x)\left(1-q^{n} y^{n}\right)\left(1-q^{n+1} x^{n} y^{n}\right)}, & 0<i<n .\end{cases}
$$

Using the functional equations relating $Z_{1}\left(s, w, \delta_{i}\right)$ and $Z_{2}\left(1-s, w+s-\frac{1}{2}, \delta_{i}\right)$ and remembering that $\left|\tau\left(\epsilon^{i}\right) / \sqrt{q}\right|=1$, we see that

$$
Z_{2}\left(s, w ; \delta_{i}\right)= \begin{cases}q^{2 s-1} \frac{1-q^{-s}}{1-q^{s-1}} Z_{1}\left(1-s, w+s-\frac{1}{2} ; \delta_{i}\right), & i=0, \\ q^{2 s-1} q^{1 / 2-s} \frac{\tau\left(\epsilon^{i}\right)}{\sqrt{q}} Z_{1}\left(1-s, w+s-\frac{1}{2} ; \delta_{i}\right), & 0<i<n .\end{cases}
$$

With this in hand, $Z_{2}$ is

$$
\begin{aligned}
q^{2 s-1} \frac{1-q^{-s}}{1-q^{s-1}} Z_{1} & \left(1-s, w+s-\frac{1}{2} ; \delta_{0}\right) \\
& +\sum_{i=1}^{n-1} q^{2 s-1} q^{1 / 2-s} \frac{\tau\left(\epsilon^{i}\right)}{\sqrt{q}} Z_{1}\left(1-s, w+s-\frac{1}{2} ; \delta_{i}\right) .
\end{aligned}
$$

When simplified, equation $(6.8)$ is the rational function for $Z_{2}$ given in Theorem 1.2 .

Acknowledgments. The first named author is partially supported by a grant from the NSF.

\section{References}

[BB06] B. Brubaker and D. Bump, Residues of Weyl group multiple Dirichlet series associated to $\widetilde{\mathrm{GL}}(n+1)$, in: [FBGH06], 115-134.

$\left[\mathrm{BBC}^{+} 06\right] \quad$ B. Brubaker, D. Bump, G. Chinta, S. Friedberg, and J. Hoffstein, Weyl group multiple Dirichlet series I, in: [FBGH06], 91-114.

[Chi08] G. Chinta, Multiple Dirichlet series over rational function fields, Acta Arith. 132 (2008), 377-391.

[CFH06] G. Chinta, S. Friedberg, and J. Hoffstein, Multiple Dirichlet series and automorphic forms, in: [FBGH06], 3-41.

[CG] G. Chinta and P. Gunnells, Weyl group multiple Dirichlet series of type $A_{2}$, in: Number Theory, Analysis and Geometry In Memory of Serge Lang (D. Goldfeld et al., eds.), Springer, to appear.

[FF04] B. Fisher and S. Friedberg, Double Dirichlet series over function fields, Compos. Math. 140 (2004), 613-630.

[FBGH06] S. Friedberg, D. Bump, D. Goldfeld, and J. Hoffstein (eds.), Multiple Dirichlet Series, Automorphic Forms, and Analytic Number Theory, Proc. Sympos. Pure Math. 75, Amer. Math. Soc., Providence, RI, 2006. 
[FHL02] S. Friedberg, J. Hoffstein, and D. Lieman, Double Dirichlet series and the nth order twists of Hecke L-series, Math. Ann. 327 (2003), 315-338.

[Pat77a] S. J. Patterson, A cubic analogue of the theta series, J. Reine Angew. Math. 296 (1977), 125-161.

[Pat77b] - A cubic analogue of the theta series. II, ibid., 217-220.

[Pat07] - Note on a paper of J. Hoffstein, Glasgow Math. J. 49 (2007), 243-255.

[Ros02] M. Rosen, Number Theory in Function Fields, Springer, New York, 2002.

Gautam Chinta

Department of Mathematics

The City College of New York

New York, NY 10031, U.S.A.

E-mail: chinta@sci.ccny.cuny.edu
Joel B. Mohler

Department of Mathematics

Lehigh University

Bethlehem, PA 18015, U.S.A.

E-mail: jbm5@lehigh.edu

Received on 27.5.2009

and in revised form on 7.2.2010

(6042) 